\title{
The Combustion and Transition to Detonation of High Pressure Flammable Gas in Closed Spaces Linked with a Narrow Path
}

\author{
Yusaku Kusuhara ${ }^{1^{*}}$, Kazuhito Fujiwara ${ }^{1}$,Fumiko Kawashima, Shingo Maeda ${ }^{1}$, \\ Ryo Nanba ${ }^{1}$
}

${ }^{1}$ Department of Mechanical System Engineering, Kumamoto University2-39-1 Kurokami, ChuoKu, Kumamoto 860-8555 Japan

*183d8457@st.kumamoto.u.ac.jp

Keywords: Combustion, High Pressure Flammable Gas, XiFoam

\begin{abstract}
When flammable gases confined or compressed in closed space such as metal cases or shells accidentally combusted, the deflagration could be generated and building up to detonation might cause intensive explosion. High energy density has been pursued in some industrial products or in some manufacturing processes, while the risk of troubles is increasing. Generally the combustion transitions to detonation in highly turbulent flows and takes some buildup time or propagation length. But in the complicated and closed space geometry such as the structure of compressors there are many interactions among compressive wave and rigid surface, and then the transition to detonation frequently has been observed. The product design considering the transition phenomena and reducing the risk of explosions is required in high energy fields.

In this study detonations of flammable gas in the high pressure vessel that has spaces linked with narrow curved path were observed and simulated numerically. A high speed camera was used to observe the flame, and the history data were acquired from pressure gauges. In the simulation, XiFoam mounted in Open FOAM was used as the base code. From the visual comparison between the results of the experiment and the simulation, it was shown that turbulent burning velocity suddenly increases and the pressure exceeds a certain value when combustion transition to detonation. These criteria is useful for the design of interior structure of high pressure facilities.
\end{abstract}

\section{Introduction}

Recently, compressors are used for various things such as air conditioners and refrigerators. The use of flammable gas as a refrigerant of the compressor is increasing. However, when oxygen and air continue to be sucked into the compressor due to human error, high temperature and high pressure air-fuel mixture is formed inside the compressor. It has been confirmed that the air-fuel mixture spontaneously ignites and a combustion explosion occurs, leading to rupture of the compressor. Therefore, it is necessary to carry out risk assessment on flammable gas.

The form of flame propagation has combustion and detonation. The distinction between them is very clear, and when the combustion wave propagates through the mixture, it suddenly transits to detonation. The combustion is characterized by a slight pressure change, while the detonation pressure rises sharply. Even when combustion occurs in the compressor, the combustion is transitioning to detonation. Therefore, a sudden rise in pressure occurs and it is considered that the compressor has ruptured. It seems that the pressure at the time of burning and the structure of the compressor are influencing the occurrence of detonation.

Therefore, in this study, we investigate the combustion of the premixed gas in the sealed high pressure container, assuming that the combustible gas burned in the compressor. As a survey 
method, a method of actually performing a combustion experiment and measuring observation and pressure, and a method of reproducing combustion by combustion analysis are used. This time we will use a model that simplifies the shape of the compressor and conduct experiments that will be the basis of future combustion experiments and combustion analysis.

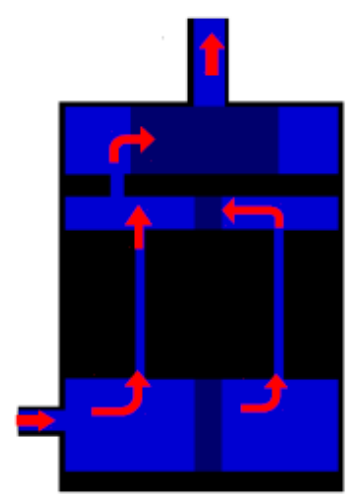

Fig. 1.1 Schematic of compressor

\section{Combustion experiment}

In order to reproduce the structure of the compressor simply, one divider was installed in the rectangular space one above the other to form a two step slit structure. The position of the twostep slit can be changed. The experimental apparatus and flow path are shown in Fig.2.1 and Fig.2.2.In order to observe combustion, acrylic is attached and visualized on the front of the equipment, and photographing is performed with a high speed camera. A pressure sensor is installed in the upper part of the flow path device, and the sensor position and ignition position are shown in Fig.2.2.

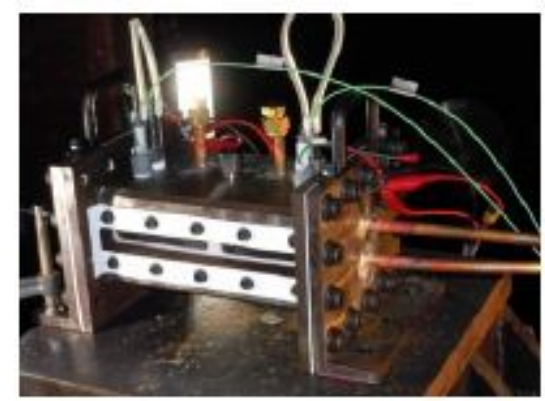

Fig.2.1 Experimental device

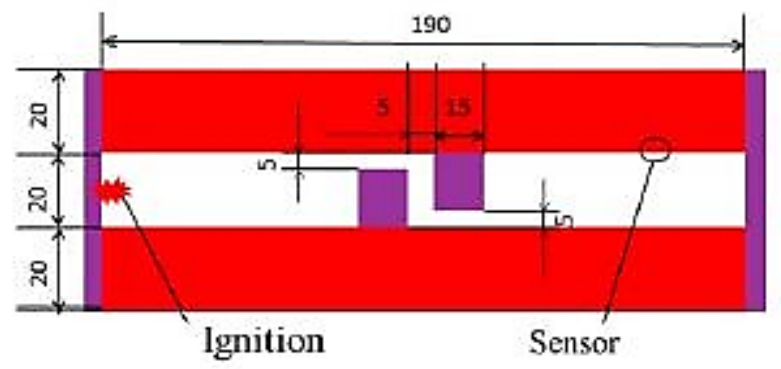

Fig.2.2 Combustion channel and position of sensors 
After filling with a mixture of propane and air (equivalence ratio: 1), ignite and burn. As an ignition method, an explosion phenomenon caused by applying an impact high current to metal thin wires is used. As the experimental conditions, experiments are carried out with a total of 4 patterns consisting of two patterns with initial pressure of $1.2 \mathrm{MPa}$ and $0.6 \mathrm{MPa}$, and two patterns with two slit positions shifted by $40 \mathrm{~mm}$ from the center and the center to the ignition side. Fig. 2.3 shows the image taken by the high-speed camera for each condition.

Table 2.1 Experiment condition

\begin{tabular}{|l|l|l|}
\hline No. & Initial pressure[MPa] & Slit position \\
\hline 1 & 1.2 & Center \\
\hline 2 & 1.2 & $40 \mathrm{~mm}$ to left from center \\
\hline 3 & 0.6 & Center \\
\hline 4 & 0.6 & $40 \mathrm{~mm}$ to left from center \\
\hline
\end{tabular}

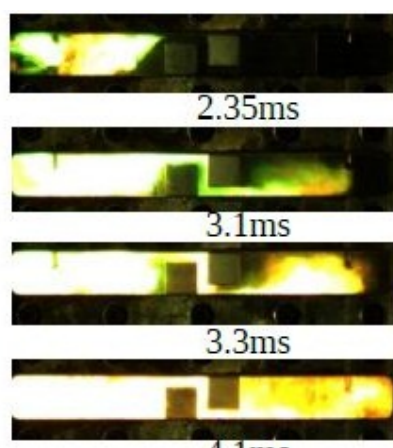

$4.1 \mathrm{~ms}$

(a) No.1

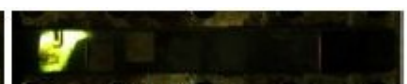
$0.85 \mathrm{~ms}$

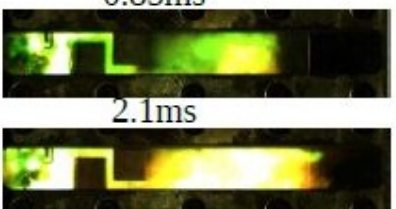

$$
2.5 \mathrm{~ms}
$$

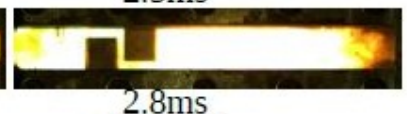

(b)

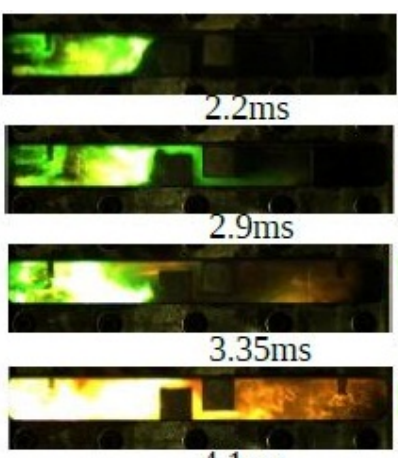

$4.1 \mathrm{~ms}$

(c) No.3

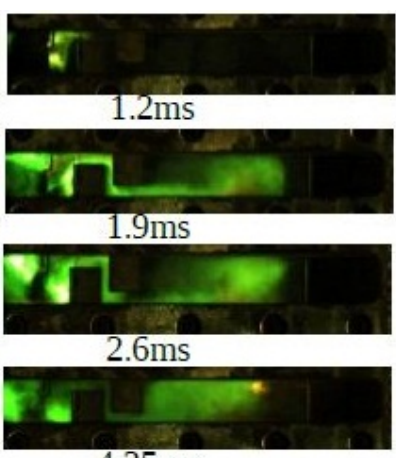

(d) No.4

Fig.2.3 Picture ofcombustion

In Nos. 1 to 3, strong combustion is occurring around the time the flame starts to spread through the two-stage slit. It is considered that transition to detonation occurs at this timing. On the other hand, in No. 4, combustion of almost constant strength is propagated and detonation has not occurred. Also, since the flame front is shaking back and forth as the combustion propagates, it was found that shock waves were generated during combustion and the reflection was repeated in the space.

Next, a graph comparing the pressure under each condition is shown in Fig.3.4.

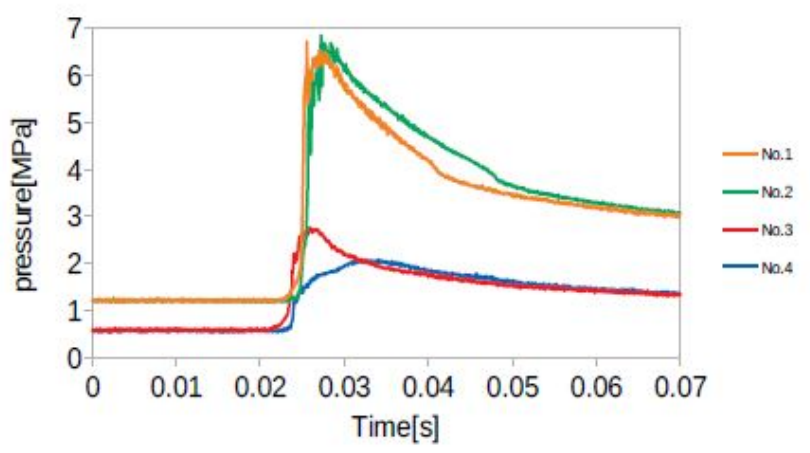

Fig.2.4 Comparison of pressure

Nos. 1 and 2 show almost the same waveform, and when detonation occurs, it is understood that the position of the slit has almost no effect on pressure. From the comparison of No. 3 and 
No. 4, it can be seen that when the detonation is occurring, the pressure rising speed increases considerably and the maximum pressure also increases. In No. 3, the time until combustion passes through the slit is long, and the pressure increases as the number of reflections of the shock wave increases in the space behind the slit. It seems that it may be easy to transition to detonation by combustion propagation to that space. From this, it is considered that safety is higher when the pressure at ignition is lower, the position of the slit closer to the ignition side.

\section{Combustion analysis}

For combustion analysis, OpenFOAM[1] which is a numerical fluid dynamics tool box is used, and XiFoam is used as a solver. XiFoam is a solver for premixed combustion considering compressibility / turbulence model[2].

The model produced what reproduced the combustible space in the combustion experiment. Four patterns of conditions similar to combustion experiments were analyzed. The model used is shown in Fig. 3.1, and the analysis conditions are shown in Table 3.1. The pressure measurement is performed at the same position as the combustion experiment.

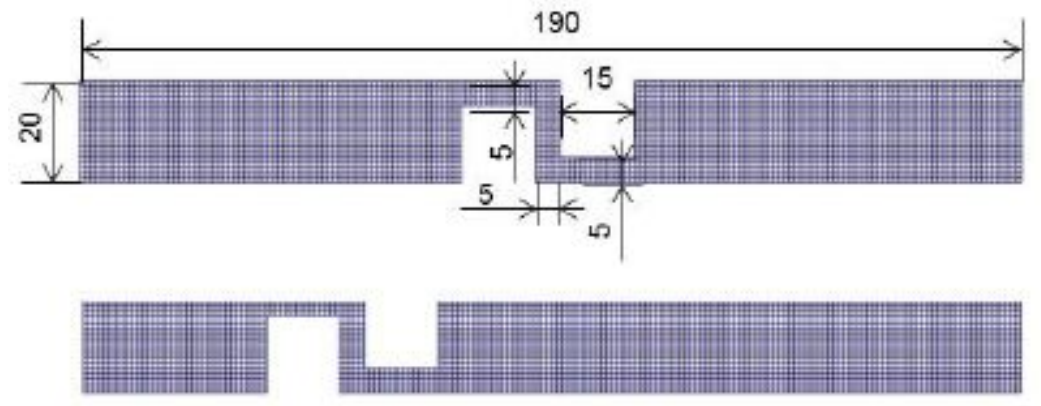

Fig.3.1 Analysis model

Table3.1 Analysis condition

\begin{tabular}{|l|l|}
\hline Use solver & XiFoam \\
\hline Mesh spacing & $\Delta \mathrm{x}=\Delta \mathrm{y}=\Delta \mathrm{z}=1[\mathrm{~mm}]$ \\
\hline Gas used & propane \\
\hline Initial pressure & 0.6 or $1.2[\mathrm{MPa}]$ \\
\hline Ignition position & $0,10,0.5$ \\
\hline Calculation time interval & $1 \mathrm{e}-6[\mathrm{~s}]$ \\
\hline Number of cells & 3350 \\
\hline Equivalent ratio & 1 \\
\hline Temperature & $300[\mathrm{~K}]$ \\
\hline Ignition source diameter & $5[\mathrm{~mm}]$ \\
\hline
\end{tabular}


Fig.3.2 shows pressure data obtained by combustion analysis by XiFoam.

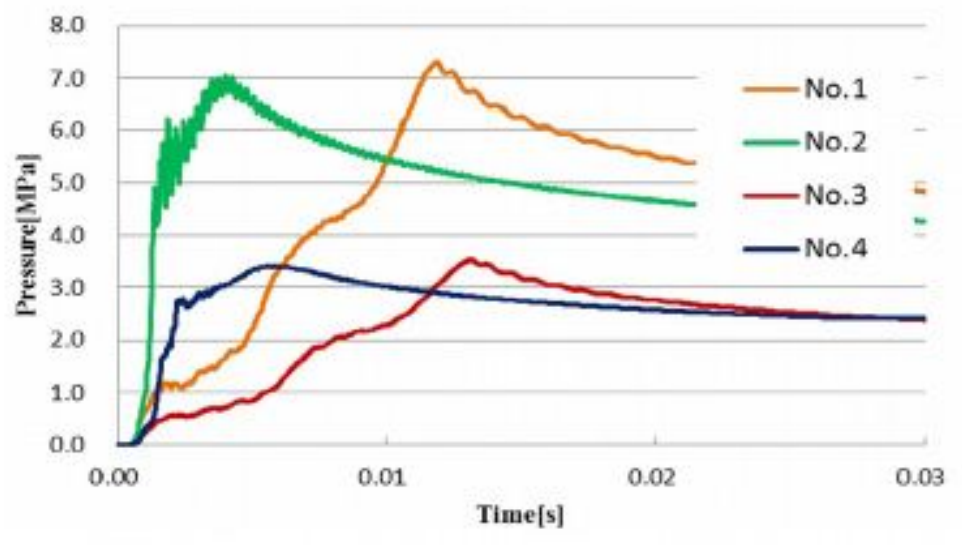

Fig.3.2 Comparison of pressure

According to Fig.3.2, in the analysis by XiFoam, there is no difference in the maximum pressure due to the position of the slit, and the maximum pressure largely depends on the initial pressure. Compared with the pressure in Fig. 2.4, when the slit position is in the middle, the pressure rise rate is smaller in the analysis.

Since the combustion that causes the transition to detonation can not be reproduced by XiFoam, investigate the transition condition and modify the solver accordingly so that the detonation can be reproduced. The turbulent combustion speed rapidly increased at any of the conditions No. 1 to No. 3, as the combustion began to spread through the slit, and in addition, the pressure increased to a constant pressure in addition all right. The temperature distribution at No. 2 in that case is shown in Fig.3.3, and the turbulent burning velocity distribution is shown in Fig.3.4.

Because conditions for transition to detonation were obtained, the condition that the turbulent burning speed suddenly rises and the pressure becomes $100 \%$ when the pressure exceeds the constant pressure is incorporated into the program of the XiFoam solver, Reproduce the transition. The values of these conditions were determined to be consistent with the experimental results.

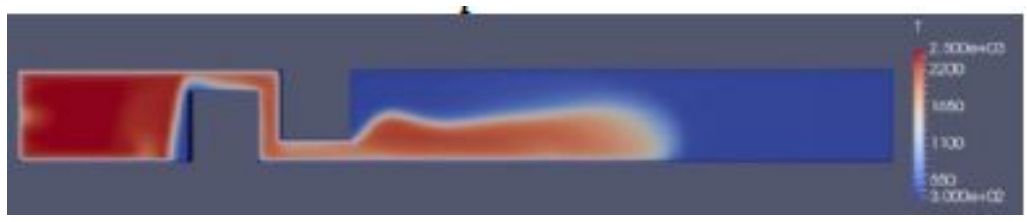

Fig.3.3 Temperature profile

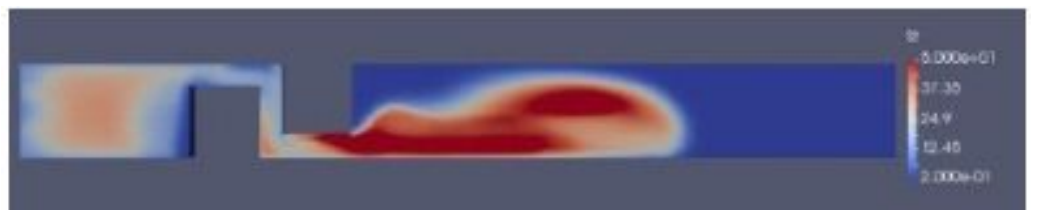

Fig.3.4 Turbulent burning velocity profile

\section{Comparison between combustion experiment and combustion analysis}

Analysis using an improved solver gave similar data in Nos. 1 to 3, so the typical temperature distribution analyzed under No. 2 is shown in Fig. 4.1, the pressure data is shown in Fig. It is shown in 4.2 . 


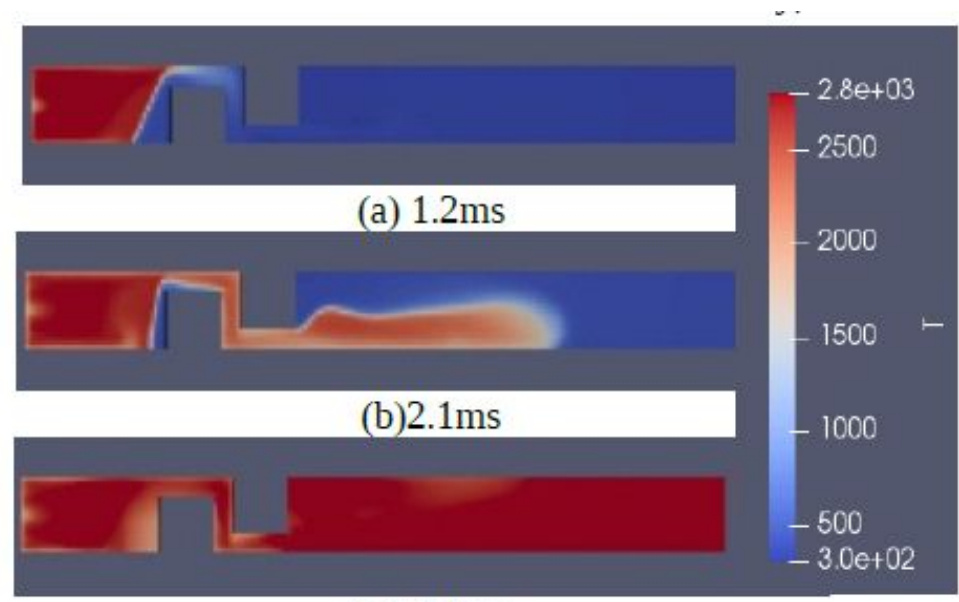

(c) $2.4 \mathrm{~ms}$

Fig.4.1 Temperature profile

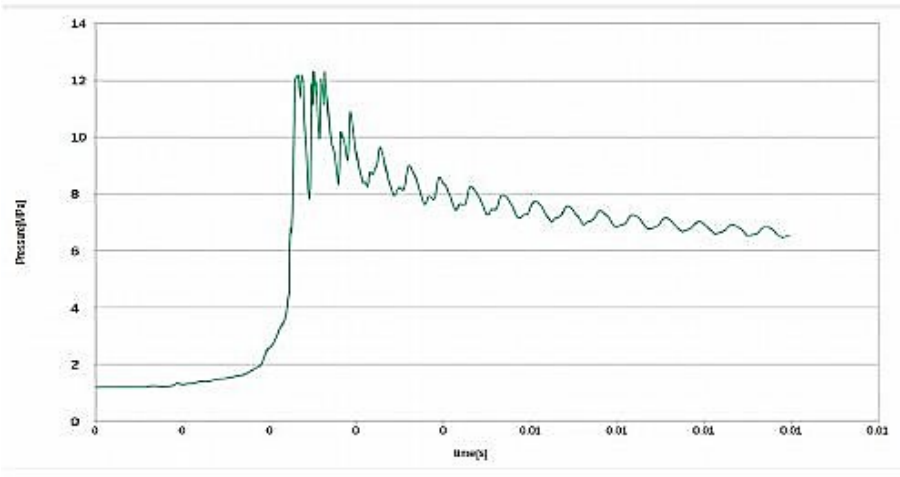

Fig.4.2 Pressure history

As you can see in Fig. 4.1, the flame starts to spread through the slit, the combustion is rapidly strengthened and the flame spreads throughout. The pressure rapidly rises after transition to detonation, rising by $20 \mathrm{MPa}$ from before the transition and the maximum pressure is about 20 times the initial pressure. Although the pressure is considerably high, it is thought that the reproducibility of detonation is high with respect to the propagation of combustion.

\section{Conclusion}

From the visual comparison between the results of the experiment and the simulation, it was shown that turbulent burning velocity suddenly increases and the pressure exceeds a certain value when combustion transition to detonation.

We developed a solver which simulates the conditions leading to combustion by detonation in a model simulating a compressor in a simplified manner and improves the reproducibility of the detonation change.

\section{References}

[1] PENGUINTIS Open FOAM information (http://www.geocities.jp/penguinitis2002/study/OpenFOAM/ OpenFOAM-info.html)

[2] Prototype Open FOAM Standard Solver List (http://dotprototype.appspot.com/OpenFOAM.html) 